\begin{tabular}{lcl}
\hline Bentham OPEN & Open Medicine Journal \\
CrossMark & Content list available at: www.benthamopen.com/MEDJ/ & $\begin{array}{l}\text { Open } \\
\text { Medine } \\
\text { lournal }\end{array}$ \\
\hline
\end{tabular}

REVIEW ARTICLE

\title{
Quality Improvement in Healthcare: Personality Type and Mindfulness as Determinants
}

\author{
Kim Peck and John Pelley* \\ Texas Tech University HSC, Lubbock, TX 79430, USA
}

Received: January 27, 2017

Revised: April 21, 2017

Accepted: July 04, 2017

\begin{abstract}
:
Background:

The emphasis on "quality" in the design of a management system for an organization was originally introduced through the work of W. Edwards Deming [1] who initially developed his QI principles to help the Toyota Motor Company increase their sales by improving the quality of their product. However, he also saw that these ideas could be applied to management of other types of organizations, such as health care organizations.
\end{abstract}

Review:

When applied to a clinical practice, QI is implemented by evaluation of structured clinical and administrative data [2], producing a "mindfulness" about QI that gives attention to several key principles: (i) focusing on data, (ii) care of patients, (iii) team problem solving, and ( iv) healthcare delivery processes that are similar for both the organization and for individual physician-patient relationship. In all instances, the effectiveness of an entire QI program is compromised if any of these principles is missing or inadequate. Such a deficiency is avoided best through a functional knowledge of personality type that increases communication at all levels. This creates a critically important organizational mindfulness for more effective QI team function and also for a more effective physician-patient encounter. The result is increased quality of outcomes at the individual patient level as well as the organizational level.

\section{Conclusion:}

The trend toward inclusion of mindfulness in healthcare develops an improved awareness of how well procedures, medications, and advice are provided.

Keywords: MBTI, Mindfulness, Quality improvement, Personality type, Physician-patient relationship, Healthcare.

\section{PREAMBLE}

In this report we wish to apply the concept of quality improvement (QI) to the physician-patient encounter. The emphasis on "quality" in the design of a management system for an organization was originally introduced through the work of W. Edwards Deming [1]. He initially developed his QI principles to help the Toyota Motor Company increase their sales by improving the quality of their product. However, he also saw that these ideas could be applied not only to manufacturing processes, but also to management of other types of organizations, such as health care organizations.

\section{THE COMPARABILITY OF ORGANIZATIONAL QI AND PHYSICIAN-PATIENT QI}

When applied to a clinical practice, QI is implemented by evaluation of structured clinical and administrative data [2]. The organization uses these data to acquire a "mindfulness" about QI. This mindfulness is produced by giving attention to several key principles that focus on:

\footnotetext{
* Address correspondence to this author at the Texas Tech University HSC, Lubbock, TX 79430, USA; Tel: (+)1806743-1116; E-mail:
} John.Pelley@ttuhsc.edu 
1. the use of relevant data,

2. the care of patients,

3. being part of a team, and

4. processes involved in the delivery of health care [3].

If we shift the focus to the individual physician-patient relationship, a comparable QI planning process also occurs following principles similar to those for the organization:

1. The physician maintains patient data that can be used to improve quality,

2. The physician maintains a focus on the care of the patient,

3. The physician functions as a member of a health care team,

4. The physician performs the processes (procedures, medications, and advice) that address illness and maintain health.

Whether applied to the organization or to the individual physician-patient, these principles function as an integrated whole, rather than independently. Thus, if any one of these principles is missing or inadequate, the effectiveness of a QI program is compromised. However, when the QI principles are used effectively, the outcome for a health care organization is improved quality, efficiency, and profitability. Similarly, when applied to the individual physicianpatient encounter, the QI principles can be seen to benefit the health of the patient just as they do for the health of the organization.

This report extends this comparison to illustrate how a functional knowledge of personality type helps to address all four principles to create a critically important organizational mindfulness. Further, when incorporated into the physician-patient encounter, this mindfulness increases the quality of outcomes at the individual patient level as well as the organizational level. We will first show how the application of personality type theory in healthcare communications profoundly enhances the quality of individual physician-patient outcomes. We will then move to the organizational level to show how this approach impacts the health care team and, finally, we will illustrate how the trend toward inclusion of mindfulness in healthcare develops an improved awareness of how well procedures, medications, and advice are provided.

\section{PERSONALITY TYPE AS A TOOL FOR QUALITY IMPROVEMENT}

Deming's work produced a theory of management that was composed of four interrelated areas. Two of these areas that have special relevance here are: 1) understanding the causes of the variation in outcomes and 2) application of psychology, especially motivation theory. Personality type as studied and identified by Jung [4] provides a mental model that explains some of the variations in perception and decision making that suggest prescriptions for improving outcomes. This area of psychology also helps us deepen our understanding of the motivation to achieve better outcomes.

\subsection{Focus on Personality Type and Communication Skills}

We can organize our thinking about personality type into two main areas: 1) differences in perception and 2) differences in decision making resulting from perception. While personality type affects numerous aspects of our behavior, the most important area in health care is in communication with patients and communication among the members of the health care team. Patient satisfaction and cooperation is determined in large part by the quality of that communication. On the other hand, most complaints about physicians relate to poor communication rather than clinical competence [5].

Fortunately, communication skills are taught in medical training to assure the clinical competency of medical graduates. The controlled environment of the medical curriculum allows learning from experience in a 'safe' environment. For example, listening skills and managing an angry patient can be practiced and improved prior to real world clinical practice. The expectations for competency in clinical practice are documented in the recently published Core Entrustable Professional Activities for entering Residency [6]. The capacity to communicate effectively with a patient is thus an expected skill on the first day of postgraduate clinical education. It would, therefore, be relevant to apply quality improvement principles to the curriculum in order to understand variation in the quality of communication skills among graduates. Deming's QI principles would then prescribe ways of reducing this variation through improvements in the curriculum. 
This raises a question as to what information and what training would be added to make a more complete education in physician-patient communication skills. A secondary concern relates to aspects of communication that are currently taught that would be displaced or might need revision with such an addition to the curriculum. It will become clear that the addition of personality type theory and practice enhances any current communication skills curriculum, and thus does not displace or modify currently taught principles. This is most easily seen when the underlying principles of Jungian personality type are understood.

\subsection{Personality Type Essential Principles}

Personality type acquires its relevance to communication because it is an unconscious mental habit that affects how a person perceives the world and how they make decisions based on those perceptions. Carl Jung observed nearly a century ago that people differed in these mental habits through simple polar opposites [7]. It was Jung that first described the familiar extravert/introvert dimension that explains how people think best: talking it out or thinking it through. Each of these behaviors becomes consistent in an individual at an unconscious level and only becomes apparent when they are required to do the opposite. Suddenly, they become very aware, and uncertain, about their behavior and they seek conditions where they can revert back to the more comfortable unconscious behavior. For example, if a person with a preference for Extraversion (E) is asked to remain quiet while they listen to an extended dialogue such as a speech or a lecture, they become uneasy. There are several reasons for this uneasy feeling, but the prime driver of the behavior is the need to think about what they are hearing. In the extravert, the energy for their thinking comes from talking as they think, i.e. you will literally be hearing what they are thinking. Clearly, circumstances that prevent the needed talking frustrate the extravert and they have to exert extra energy to cope. This uncomfortable situation is often relieved with self-talk. The extravert will resort to holding a silent conversation in their heads under circumstances where they cannot speak.

This concept is extended to a person with a preference for Introversion (I) in a different way because the comfort zone is to think silently before speaking, if speaking is needed at all. If the introvert is asked to speak about an issue before they can fully process it silently, the result is awkward and halting sentence structure and muted volume. The emotional aspect of this outward behavior further complicates communication through embarrassment. Both examples of introvert and extravert illustrate several important aspects of Jungian personality type:

1. Personality type is about normal behavior. There are no pathological aspects to any of the type preferences. Thinking to yourself is a normal preference and thinking out loud is a normal preference. There are no good or bad types, no sick or well types, nor are there smart or dumb types. Jungian personality type is only a preference, not an outcome.

2. Everyone uses both their preference and the opposite to their preference every day. This is because people must adapt their behavior to their circumstances. It is the various circumstances that change throughout the day, not the preference known as personality type. This is a critical concept because it teaches that people are adaptable and not rigidly constrained by their type preference.

3. Whenever a person has to perform in their opposite, they use extra energy and as a consequence they will become fatigued sooner. An introvert who teaches by giving a lecture will be much more fatigued at the end of the lecture than an extravert. In fact, the extravert will likely be energized and want to continue. The need to communicate with a patient of opposite type will have the same effect.

4. Personality type is a persistent tendency throughout life. Some people mistake changing scores on the MyersBriggs Type Indicator, an instrument that is used to identify type [7], as a real change in their personality. What is actually changing is an increased ability to adapt by using the preference opposite as a skill. Early attempts to identify type can be biased by a number of factors including expectations of family and friends, self-concept, and simply mistaking what they do for what they prefer. Maturation usually allows greater self-knowledge and removes much of this bias.

5. People of the same type use the preferences very individually. Type only gives the general tendency to choose, or use, one of the two opposites preferentially but they do this in their own way. Extraverts only have in common the desire, when they have the choice, to think out loud. What they think about, how well they think, and how they express their thinking will be their personal individuality. But, if you want to know what they are thinking, all you have to do is listen. By contrast, if you want to know what an introvert is thinking, you need to ask. 
It is the capacity to adapt behavior to that of the opposite and to use that as a skill that allows the knowledge of type to be used as a tool for communication. The awareness that use of the opposite uses more energy, that the opposite can be developed, and that personality doesn't change because of external factor eliminates potential attitude barriers to using personality insights to apply QI principles to patient communication.

\subsection{Personality Type Dimensions}

There are three other dimensions that compose personality type, all of which are relevant to the physician-patient interaction: 1) Sensing vs. Intuition, 2) Thinking vs. Feeling, and 3) Judging vs. Perceiving.

1. The preference for Sensing $(\mathrm{S})$ versus its opposite, the preference for Intuition $(\mathrm{N})$ is applied to the way people tend to take in information, i.e. to what do they give their attention. This focus on the type of awareness that people exhibit caused Jung to refer to them as preferences in perception.

2. The preference for Thinking ( $\mathrm{T}$ ) versus its opposite, the preference for Feeling (F). These are applied to the way people reach decisions about what they perceive, i.e. how do they prioritize their choices. The need to weigh what is perceived against established criteria, either logical or emotional, caused Jung to refer to them as preferences for judgment.

3. The preference for Judging $(\mathrm{J})$ versus its opposite, the preference for Perception $(\mathrm{P})$ was not one of the original Jungian observations. Instead, while her Myers-Briggs Type Indicator (MBTI) was under development Myers discerned that people manage their time with opposite preferences. She identified those who emphasized Jung's preferences in judging, $\mathrm{T}$ and $\mathrm{F}$, as Judging types and those who emphasized the preferences in perception, $\mathrm{S}$ and N, as Perceptive types. Since Judging types constantly evaluate their situation they tend to live by a plan. Their opposite, the Perceptive types are constantly seeking new information so they tend to be adaptable and flexible.

Jung applied the term "Sensing" to those people who gave their attention through their five senses: vision, hearing, touching, smelling, and tasting (Although balance is also a sensory modality, Jung did not give his attention to it as with the others.) When one relies on the senses, it puts their attention in the present and, as a result, the sensing type can be characterized by the question phrase, "what is?" The present, however, is also a function of the past. What we see on a regular basis is largely a function of our memory of that sight in the past: a familiar room, route to work, or coworkers. The retina primarily provides the aspects of vision that have changed or are different from what is remembered. This leads to a frequent comment from neuroscientists that "we see with our brain." Taken together the sensing type gives most of their thinking time to the present and the past. They are detail oriented and are driven to seek certainty in the accuracy of their memory and/or in how they perform a procedure. A sensing type patient will be more concerned with step by step instructions. They will ask "what" and "how" questions, and they will want precise descriptions. They will not be interested in understanding their problem, just what to do about it.

Similarly, Jung applied the term "Intuitive" to those people who gave their attention to future possibilities. The creation of possibilities requires an integration of what is known already to create questions about how things might be related. It is an anticipatory state of mind that tolerates trial and error. In students, this preference drives learning toward forming a "big picture" often at the expenses of learning details. Because of this future orientation, the intuitive type can be characterized by the question phrase, "what if?" An intuitive type patient will therefore be concerned with both current and long-term implications. They will also ask "why" questions in order to understand their situation and, instead of focusing on details, they will talk more in generalities.

The "Thinking" type was named by Jung to describe those people that reached decisions through logic. This requires weighing evidence objectively and independently of how others may have reached their decision. In a patient, there is little attention given to how the physician "feels" about them, just that the physician make sense. This type of patient will tend to "test" the physician's advice or knowledge.

The "Feeling" type was named by Jung to describe those people that reached their decisions by referring to their values. This is not a reference as much to biological emotion as it is to maintaining a rational priority based on quality of life. It is a common mistake to consider thinking types to be an opposite preference due to having no emotion and this mistake is easier to avoid by focusing on what the person trusts. Thinking types trust their logic more than their emotions and feeling types trust their emotions more than their logic. 
The "Judging" type was named by Myers to describe those people who emphasized decision-making in the way they managed their time. They operated from an awareness of a schedule or "to-do" list and were happiest when tasks were completed on time, i.e. they sought the "joy of closure." Judging types tend to be driven to closure and risk making a decision too soon.

The "Perceptive" type was named by Myers to describe those people who emphasized discovery in the way they managed their time. They operated from an awareness of making decisions based on gathering information, even at the expense of extra time. They likewise use a "to-do" list, but more as a record of what needed to be done. They were not as aware of deadlines and are not as bothered by postponing tasks, i.e. they sought the "joy of discovery." Perceptive types tend to be driven to gathering information and risk a long delay in making a decision.

\subsection{Preferred Communication Styles}

Communication is the most effective between two people when they have similar communication styles [8]. Their thinking is shared with clarity and harmony when both perceive the world the same and when both make decisions the same. For example, couples are more satisfied in their relationship when their personality type matches, or when they can easily adjust to that of their partner. This has been shown to also hold in the health care field through a critical incident analysis that compared the quality of the communication against their verified MBTI type [8, 9]. In this study which followed a standard question protocol, both patients and health care professionals were asked to describe both a satisfying clinical encounter and one that was dissatisfying. The protocol asked participants to comment on the nature of the encounter, who was involved, what they were thinking or feeling about the situation, what was actually said, and what happened as an outcome. These narratives were matched against the type of the physician and the patient to establish consistencies with their verified MBTI type. This led to a communication model that incorporated the effect of the patient's personality type [8]. As shown in Table 1, there are three stages of interaction with the second stage having some remarkable findings:

Table 1. Behavior cues in the patient*.

\begin{tabular}{|c|c|c|}
\hline Stages of Interaction & \multicolumn{2}{|c|}{ Patient's Type Mode } \\
\hline Beginning the interaction & $\begin{array}{l}\text { Talk it out } \\
(\text { E - Extraversion) } \\
\text { - Action and animation } \\
\text { - Direct eye contact } \\
\text { - Louder tone } \\
\text { - Faster pace }\end{array}$ & $\begin{array}{l}\text { Think it through } \\
(\mathrm{I} \text { - Introversion) } \\
\text { - Measured action } \\
\text { - Averted eye contact } \\
\text { - Quieter } \\
\text { - Slower, more considered pace }\end{array}$ \\
\hline \multirow[t]{2}{*}{$\begin{array}{l}\text { Investigating needs } \\
\text { and } \\
\text { planning action }\end{array}$} & $\begin{array}{l}\text { Specifics/Logical Choices } \\
\text { (ST - Sensing and Thinking) } \\
\text { - Values facts } \\
\text { - Provides facts in logical order } \\
\text { - Practical approach to completing task } \\
\text { - Wants tried and tested methods }\end{array}$ & $\begin{array}{l}\text { Specifics/Impact on People } \\
\text { (SF - Sensing and Feeling) } \\
\text { - Values personalized service; caring relationship } \\
\text { - Prefers facts to theory } \\
\text { - Wants warm, friendly approach } \\
\text { - Wants what worked for others }\end{array}$ \\
\hline & $\begin{array}{l}\text { Big Picture/Logical Choices } \\
\text { (NT - Intuition and Thinking) } \\
\text { - Wants logical options } \\
\text { - Tests physician's competence } \\
\text { - Values cutting edge methods }\end{array}$ & $\begin{array}{l}\text { Big Picture/Impact on People } \\
(\mathrm{NF} \text { - Intuition and Feeling) } \\
\text { - Views their situation as unique } \\
\text { - Needs to be valued as a whole person (holistic) } \\
\text { - Interested in new methods }\end{array}$ \\
\hline Establishing closure & $\begin{array}{l}\text { Joy of Closure } \\
(\mathrm{J} \text { - Judging) } \\
- \text { Values the goal } \\
\text { - Moves dialogue to closure } \\
\text { - Impatient }\end{array}$ & $\begin{array}{l}\text { Joy of Discovery } \\
(\mathrm{P} \text { - Perceiving) } \\
\text { - Values path to goal } \\
\text { - Easy with adaptive approach } \\
\text { - Side tracks with issues during dialogue } \\
\text { - Easy with last minute action }\end{array}$ \\
\hline
\end{tabular}

*Adapted from Allen 2000.

1. Meeting or beginning the interaction

2. Investigating needs and suggesting actions to be taken

3. Establishing closure.

The findings showed a correlation between the descriptions of each stage of the clinical encounter. The beginning interaction showed behavior cues that matched the persons MBTI type for Extraversion and Introversion. The findings for the second stage that investigated needs were remarkable in revealing that the behavior cues of the patient became 
ambiguous unless all four possible combinations of type preferences for Perception and Judging, i.e. ST, SF, NT, and NF were considered. In the course of the study as will be detailed below, these combinations also defined four individual ways that patients prefer to receive bad news. The finding that is more relevant to the physician, or the teacher of communication skills in the medical curriculum, is that the cues can be learned and identified during the normal course of the interaction. This obviates the need for extra data collection prior to the visit. However, as the situation dictates, it does permit a more informed entry into the patient chart concerning the quality of the dialogue. Some electronic records contain a scripted sentence that can be added to the medical record under a menu item that states "Education Response: Verbalized understanding, Understanding confirmed by return demonstration, Communication with the patient (or representative) occurred in a manner that met their communication and health literacy needs." This permits recording of impressions of clarity and agreement with the treatment plan or uncertainties that need a follow up at the next visit. Workshop materials are commercially available (www.capt.org) to train physicians and their health care staff in adapting their communication style to fit that of the patient.

\subsection{Breaking Bad News}

One of the foremost QI issues in health care communications is the way that bad news is delivered. To be sure, there are established protocols and advice given in every medical curriculum, but these protocols assume all patients received the information in the manner most comfortable to them. Compounding the problem, all patients are similarly treated as if their participation in the decisions was the most comfortable to them. The research shows that, if the patient's MBTI type matches that of the physician, this difficult conversation will go as well as possible with both parties unaware of the reason why. The QI complications come in when there is misunderstanding or other discomfort and the reasons remain a mystery. To appreciate the dramatic differences, and relatively straightforward adaptations required of the physician, excerpts of comments by patients are presented in Table 2.

Table 2. MBTI type preferences for receiving bad news in the clinical encounter*.

\begin{tabular}{|c|c|}
\hline \multicolumn{2}{|c|}{ Patient's combined preference for perception and judging: Excerpts from comments about satisfaction with receiving bad news. } \\
\hline $\begin{array}{l}\text { Plain Facts with Practicality } \\
\text { (ST - Sensing and Thinking) } \\
\text { - I Just want to hear the facts; nothing irrelevant." } \\
\text { - They kept asking me how I felt; it felt intrusive. I won't open up until I } \\
\text { trust you. } \\
\text { - No sugar coating. }\end{array}$ & $\begin{array}{l}\text { Personal Service with Kindness } \\
\text { (SF - Sensing and Feeling) } \\
\text { - He was too blunt; it upset me. It helped a lot when the nurse was kind } \\
\text { to me. } \\
\text { - I had to hear the news without my partner here. } \\
\text { - It was helpful to not be hurried and have his full attention. }\end{array}$ \\
\hline $\begin{array}{l}\text { Logical Alternatives with Competence } \\
\text { (NT - Intuition and Thinking) } \\
\text { - Respect my need to understand. } \\
\text { - Respect my intelligence and don't hide anything. } \\
\text { - Demonstrate your competence. } \\
\text { - Give me all the options so I can see any patterns. }\end{array}$ & $\begin{array}{l}\text { Supporting My Vision } \\
(\mathrm{NF} \text { - Intuition and Feeling) } \\
\text { - Treat me as a whole person; with respect. } \\
\text { - Value my concerns; listen to me. } \\
\text { - Provide an overview so I can see any overall solutions } \\
\text { - Take time to discuss my concerns; and be honest! }\end{array}$ \\
\hline
\end{tabular}

\subsection{Summing Up Personality Type Awareness and QI Principles}

The available evidence concerning Jungian type as determined by the MBTI indicates that differences in the type preferences, especially those for either Sensing or Intuitive perception and Thinking or Feeling judgment, are significant factors in establishing quality in healthcare delivery. All four principles of QI planning can be fulfilled with this new awareness. An awareness of the patient's presenting type as determined through behavioral cues can become part of the medical record, the enhancement of communication with the patient has a direct effect on the quality of care, and the necessary skills to implement this new data set can be learned through training that is already available. The fourth principle is that of maintaining a quality interaction as a member of the health care team. In the next section, we describe how personality type insights facilitate communication in team problem solving to aid healthcare QI teams.

\subsection{Personality Type And Healthcare QI Teams}

Apart from the physician-patient interaction, a second venue for implementation of QI principles in the health care organization is the healthcare team. At its core, QI is a team process [3]. The strength of the team over separate individuals is that it harnesses the knowledge, skills, experience, and perspectives of each individual on the team in order to make strategic improvements. 


\subsection{QI Operations that Require a Team}

The HRSA Quality Improvement guidelines indicate that a team approach is needed in virtually all healthcare QI operations. These guidelines specify that a team is needed when:

\subsubsection{The System is Complex}

Healthcare organizations function as complex adaptive systems [10] that are characterized by the non-linear relationships between their components. The result is that cause and effect are usually separated widely in time and surprise and uncertainty are constant elements that challenge attempts to implement policies. The need for team problem solving has been proposed as one of five disciplines needed for organizations to learn [11].

\subsubsection{No One Person in an Organization Knows all the Dimensions of an Issue}

Even the top level of management is limited by the quality of information received. As the environment changes, the need to accurately perceive reality increases.

\subsubsection{The Process Involves More than One Discipline or Work Area}

Healthcare has never been an individual sport. While the responsibility for higher order thinking involved in diagnosis and determination of a treatment plan is a central role in health care, this role alone is rendered impotent without the availability of the remainder of the healthcare team.

\subsubsection{Solutions Require Creativity}

The health care organization is not alone as a complex adaptive system. It operates in an environment that is also a complex adaptive system an order of magnitude greater. This requires more than adherence to best practices; it requires development of new best practices.

\subsubsection{Staff Commitment and Buy-in are Needed}

It is a long held management proverb that people support best, that which they help to create. It is not enough to create a solution and expect it will automatically work. Those implementing a new policy must also believe in it and be motivated to overcome barriers.

In all five of these situations, teamwork is the only viable route to the learning needed to solve complex problems. This is because all learning is conversational. This also applies when the problem solving scenario is extended from a physician-patient interaction to a group or team interaction. It is still incumbent on each member of the group to contribute to a feasible solution, i.e. a treatment plan for the organization. As such, problem solving depends on precision in communication between team members just as it does between physician and patient. It is well established that the same principles of personality type that can aid physician-patient communication can also apply to team communication.

\subsection{Team Dynamic vs Physician-Patient Dynamic}

In order to apply personality type concepts to teamwork, it is necessary to first compare the team to the dynamic involving the physician and patient. The counterpart for the patient in a QI program is the organization itself. Organizations exhibit both health and disease. When they are healthy, organizations demonstrate:

1. Adaptability to change. An effective healthcare team will detect the need for change through evaluation of the QI data that is collected. Since adaptation involves action, a team must create alternative actions and reach consensus on the best one.

2. A sense of identity. Since commitment and buy-in throughout the organization is critical, a team must include in their problem solving conversation the sense of mission and goals shared by all members of the organization they represent.

3. A capacity to test reality. The nature and quality of the data must realistically and accurately reflect both the environmental factors and the reality within the organization. Just as a patient in denial is unlikely to follow a treatment plan, the organization in denial cannot solve its QI problems.

4. An integration of component parts. Teams are obliged to assume a holistic perspective in their formulation of any 
QI issues or problems suggested by the data. Any attempt to solve localized problems within the organization will have a high likelihood of "side-effects." Solving a problem for one component of the organization can create a new problem that leaves the system, the health care organization, unhealthy in a different way. An example at the patient care level would be prescribing a beta-blocker for hypertension only to create sexual dysfunction. A team with multiple perspectives is more likely to anticipate side effects within the organization because each personality type brings a new and balancing perspective.

\subsection{Personality Type Contribution to Development of Team Cohesion}

Teams undergo an obligatory developmental process that mirrors the growth and development of a person's psyche. Overall the team progresses from its conception through stages that first manifest the id, followed by the acquisition of an ego, and ultimately development of the more complex and competent superego. The id is the stage where the individuals on the team find the interaction with others members to be comfortable only when their personality types match. This correlates with the selfish and wishful nature of the id. Alternate personalities are a threat and, even in the best case, require extra energy to understand and achieve consensus. At first, each member of the team is behaving normally within their own id. The process of learning to adjust and accommodate is a normal response by team members who are problem oriented. This adjustment to the reality of working together, guides the development of the team's "ego" both through the satisfaction that comes from consensus and through the discomfort that comes from discord. The team, then, develops a healthy ego by accepting responsibility for social realities and norms. One aspect of reality that the team acquires is the development of a vision that extends to values and mission beyond the team itself, in this case to the organization. This vision of the ideal "self" for the team indicates the development of the team's superego. It provides the team with aspirations and values that transcend that of each individual member. The superego is the counterpart of all personality types embracing the thinking of their opposite types and offering, in turn, the gift of their own type preferences.

This psychoanalytic model of team development is supported by a developmental sequence described by Tuckman [12]. He proposes four stages in group development: 1) forming, 2) storming, 3) norming, and 4) performing. The initial stage of forming is a process of the team learning about each other. They think more about each other than about a problem. As competition for roles in the group intensifies, the uncomfortable necessity to adapt to alternative points of view develop. This storming stage brings to mind the ego undergoing development as the need to adapt to reality increases. The clash of opposite preferences in others on the team poses a challenge to each team member to understand and accept alternative points of view. As the team matures it begins to show the first signs of cohesion characterized by the development of trust. In this norming stage, they are no longer a collection but a community. Thinking is improved because listening and understanding are improved. At this point the team will now spontaneously move to communicate in a way that optimizes their synergy. The QI team is now performing, focused more on the situation than it is on the rules. Both the efficiency and the effectiveness with which teams progress to this point will be greatly affected by how well they grasp a basic understanding of personality type.

\subsection{Role and Interaction of Personality Types in Team Dynamics}

Each dimension of the MBTI types contributes to team dynamics. When team members understand that preferences are based in normal behavior, they no longer suspect that there is "something wrong" with another team member. They also don't overvalue a team member that has their same personality type. Just as we have seen in the stages of interaction between a physician and patient (Table 1), personality type will influence the development of team cohesion and contribute to producing a highly performing team.

The role of the extraversion and introversion preferences determine how the team members prefer to think. The Extraverts will energize the team by their need to think out loud. They will keep the dialogue and thinking active among all team members. The Introverts, by contrast will act more as compilers. They will listen and process information, and most importantly they will provide depth of thought. Once each accepts the other for their respective roles the team will have acquired both interplay and depth of thought. They will value both speaking up and thinking deep.

The role of sensing and intuitive preferences determine how the members manage the data. The Sensing types will serve more as information resources with their attention to the details of the data. They help Intuitives pay attention to important data. The Intuitives will serve as information integrators to help sensing types see relationships and correlations. The Thinking types will make sure that relationships and conclusions make sense; they serve as testers to keep the group relevant. The Feeling types will help the group through moments when the thinking gets stuck or 
coming to consensus is difficult. Their tendency to harmonize reminds the group of the values they seek and the goals for quality improvement.

The pace of the conversation is balanced by the judging and perceptive preferences. The Judging types will help to seek closure through consensus and, thus, they will act to keep the group on task. The Perceiving types will help seek the discovery of either more data or the creation of more alternative solutions. Once they are aware of the value of discovery, the Judging types will tend to be more patient with the Perceptive types, but they provide, in turn, an essential awareness of time constraints. Similarly, once they are aware of the role of Judging types in moving to closure, Perceptive types, will use their thinking time more judiciously.

The awareness of the thinking and behavior of opposite preferences helps a team grow through the forming and storming stages efficiently. However, a protocol that make the best use of type differences can enhance the process even further. This protocol has been termed Zigzag Problem Analysis [13] and it provides any team with an effective place to begin.

\subsection{Putting it Into Action}

The Zigzag Problem Analysis process was derived from the observation that four of the type preferences described mental functions in information processing. These mental functions are sensing, intuition, thinking and feeling. The Zigzag term was applied due to the established representation of type dimensions such that a line drawn through the mental functions in the order listed created the letter "Z" Fig. (1). The procedure for conducting Zigzag Problem Analysis is as follows:

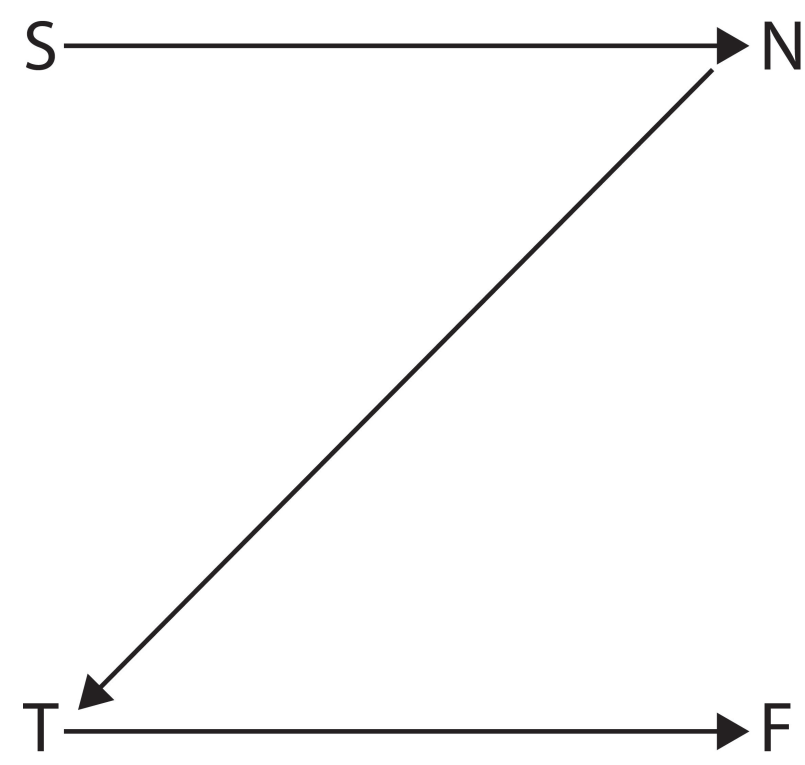

Fig. (1). Zigzag problem solving.

1. Focus on the sensing function. The team seeks answers to questions like the following:

a. What are the available data?

b. Have the data been analyzed?

c. Have the data changed over time?

d. What has been done?

e. What other data are needed?

There is a conscious effort to avoid consideration of alternatives, logical conclusions or subjective considerations, in other words, the other mental functions. With experience the group might refine the nature of the questions, but the only focus is on the data. The Sensing types are aware during this step that they have the edge and that their contribution is critical. The intuitive members are unconsciously developing their abilities to be aware of the data. 
1. Focus on the intuitive function. The team seeks answers to questions like the following:

a. What do the data imply?

b. What data are the most important?

c. What are the alternative solutions or approaches?

d. Is this problem analogous to another similar one?

There is now a shift in focus to the creative process of developing alternative solutions. Any effort to judge the alternatives is postponed to the next step. The group maintains the intuitive mode to expand alternative solutions based on the data examined so far. The intuitive members are now at their best and the Sensing types are unconsciously acquiring intuitive abilities.

1. Focus on the thinking function. The team seeks answers to questions like the following:

a. What data are missing?

b. What are the pros and cons?

c. What are the logical consequences of each solution?

d. What is the cost of each solution?

The focus on logical evaluation of the alternatives puts subjective issues aside. Hard data demand hard decisions. The group begins the process of analyzing each alternative for strengths and weaknesses in order to prioritize them. The members with a thinking preference are now at their best and they help to avoid digression into subjective issues. The Feeling types are unconsciously developing a greater capacity for using logic, but are having difficulty separating the blunt objective dialogue from their own need to prioritize their own personal values.

1. Focus on the feeling function. The team seeks answers to questions like the following:

a. How much do people care about the outcome?

b. How much does each alternative affect quality improvement?

c. Who will be committed to the outcome?

d. What and whose values are involved?

e. How will patients and their families react to each of the alternative solutions?

The focus on feeling evaluation of the alternatives calls on the whole group to extend their objective thought to the subjective aspects. The Feeling types bring out complexities that are not revealed by logic while the Thinking types unconsciously develop a sense that any logical conclusion is likely to have human implications.

While this Zigzag protocol gives structure and efficiency to a team, it nevertheless requires an awareness by each of the team members of the characteristics of each of the other members. This requires an organizational step prior to beginning the first step of the protocol. There are several variations that could be used to help team members become familiar with each other. Each member can report their own personality type including how they think they can help the team. Examples, if available, will help to develop familiarity at a personal level. Members can also report on how their opposite will be helped by th ose on the team who have those mental functions as preferences. After a discussion that has included each member of the team, understanding replaces tolerance. When understanding becomes established it is possible to develop the primary characteristic observed in all higher order thinking - mindfulness.

\subsection{Mindfulness And Deliberate Practice}

The discussion of personality type in both physician-patient encounters and in problem solving teams illustrates that an awareness of the influence of the different personality type preferences is critical. This critical awareness is, in itself, a skill that must be deliberately developed through life-long practice. This may come as a surprise, since simply calling attention to awareness could be considered to be enough. It would be enough, if it were not for distraction. However, distraction created by the changing stimuli in our daily tasks prevails. This will be as true for QI practice as it is for any other task. A physician who remains preoccupied with a prior diagnostic or treatment conflict, will not maintain the awareness needed to use personality type as a tool in communicating with the present patient. More generally, any member of the health care team who unconsciously automates their behavior in their daily tasks will likely overlook important elements of QI policy. An example would be a physician's assistant who overlooks drug interactions in the history that needs attention by the attending physician. Scanning the pharmaceutical history for dosages and matching against findings from the physical exam or laboratory tests is standard practice, but it takes an extra awareness to 
compare drug interactions. Other case examples could be drawn from patient handoffs in the transition of care or the accuracy and completeness of entries into the medical record.

\subsection{Awareness as a Skill}

It is fortunate that not only is awareness critical to skill development, it is a skill in itself. Awareness of weakness has been studied as an essential factor in skill development through deliberate practice [14]. The concept of deliberate practice uses awareness of weakness to determine the practice needed. Awareness as a skill has been studied in the emerging field of mindfulness research. The practice of mindfulness meditation has been shown to positively affect the frontal cortex of the brain in a way that improves the ability to focus [15]. The frontal cortex area is significant in our consideration of QI since it is the same area of the brain that is engaged in data analysis, problem solving and decision making.

The research on deliberate practice has been driven by evidence that superior skill is not correlated with inherent talent or with intelligence, but instead with the use of a form of purposeful, focused practice that Ericsson termed "deliberate practice." While deliberate practice emphasizes the characteristics of the learner, it also describes the role of a teacher that is needed to help assure that the practice is also "perfect practice." However, it is encouraging that in the long run, once the learner has acquired competency, a teacher is no longer needed and is replaced by self-teaching. While the concept of becoming an expert has not been emphasized as a concern in QI practice or problem solving, a closer look reveals that it should be.

\subsection{Awareness in Deliberate Practice}

Deliberate practice is a focused effort designed for the purpose of improving performance. It is most effective in the early learning stages under a teacher's guidance so that the teacher can define what needs to be improved. If a physician is trying to learn how to adapt their dialogue to match that of their patient's personality, a teacher can not only detect where improvement is needed, but also show how to improve. Deliberate practice requires substantial repetition, but also repetition with awareness. A member of a QI team must likewise be continually aware of the way those of opposite personality type will express themselves.

Research studies on physicians have tested the traditional theories that experts have automated their behavior. The implication is that the expert functions spontaneously. This would indicate that the use of a tool like personality type would be acquired through practice, such as deliberate practice, and then subsequently be guided by an unconscious instinct. However, studies of several aspects of physician performance subsequent to training show that this is not the case. Instead, skills deteriorate over time when their performance becomes automated. It is a natural function of the brain to automate behavior in order to devote energy to new demands. However, automation precludes awareness due to its robotic nature. This creates a challenge for all those who help fulfill the responsibilities of the health care team. They need to develop the skill of maintaining awareness in an atmosphere of repetition. The implications, then, are that both QI planning and QI practice require continual skill development. A method for developing awareness as a skill, that has been gaining increased attention is mindfulness meditation.

\subsection{Mindfulness Practice and Maintaining Awareness}

The healthcare environment is a multi-tasking environment. By extension, a QI program will always function in a multitasking environment making it essential that all members of the healthcare organization have developed awareness as a skill. The awareness needed is not an attention to many tasks at once, but rather an attention to "task switching" [16]. Thus, the skill is one of switching awareness. Studies on media multitaskers has shown that practice with simple breathing exercises improved their capacity to switch their attention [17]. Thus, the path to an effective QI program is made more certain with the regular practice of mindfulness exercises. One of the earliest and simplest approaches to establishing and maintaining health was described by Benson in The Relaxation Response (1975) [18] and new approaches continue to be published [15]. This is an emerging area of research in health care ranging from applications in the treatment of anxiety and depression and emotional regulation [19], substance abuse [20], and somatic conditions such as pain [21].

The needed awareness of the effect of personality type in communications benefits both patient care and QI problem solving teams. When the members of the healthcare organization develop and maintain their skill of awareness through the practice of mindfulness meditation, they increase the likelihood of applying personality type as a tool. 
Table 3. Members of the healthcare team.

\begin{tabular}{|l|}
\hline 1. Doctors \\
\hline 2. Physician assistants \\
\hline 3. Nurses \\
\hline 4. Pharmacists \\
\hline 5. Dentists \\
\hline 6. Technologists and technicians \\
\hline 7. Therapists and rehabilitation specialists \\
\hline 8. Emotional, social and spiritual support providers \\
\hline 9. Administrative and support staff \\
\hline 10. Community health workers and patient navigators \\
\hline
\end{tabular}

\section{SUMMARY}

Early studies on quality improvement (QI) in manufacturing revealed an opportunity to apply the same principles to the delivery of health care. When applied to clinical practice, QI is implemented with data collection practices that measure healthcare outcomes. When a QI planning process is implemented, a mindfulness about the health care delivery process is developed. This report extends the established QI process from the organizational level to the physician level with a specific focus on improvement of quality healthcare through the use of insights from personality type theory. Studies on patient satisfaction as a function of their MBTI personality type reveal a positive correlation with patient satisfaction when the physician is able to adapt their dialogue to reflect that of the patient's personality type. The conclusion is that personality type represents a communication "style." An awareness of the patient's presenting type as determined through behavioral cues can become part of the medical record and eventually used as QI data. The same principles that enhance the quality of the physician-patient interaction can be applied to the interaction of members of a healthcare QI team. The interaction of team members requires clear communication that takes the different personality types of the team into account. Recommendations from the QI team must take a complex health care organization replete with uncertainty and surprise into account. This demands skill in gathering the best data by the Sensing type team members and by interpreting that data by the Intuitive type team members. A Zigzag Problem Analysis process uses each of the type preferences in a defined order to maximize the use of time and to organize the discussion.

The successful use of personality type insights in the physician-patient interaction and in problem solving by the QI team demands constant awareness. The ability to maintain this awareness is a skill that can be learned, but it must also be continually practiced. The research on skill development shows that the deliberate practice of these skills under a teacher's guidance during training, preferably in their formal professional education, allows the continued refinement of these skills throughout their careers. Research findings with mindfulness meditation indicate that the regular practice of focused relaxation trains the brain to maintain focus in a multitasking healthcare environment.

\section{CONSENT FOR PUBLICATION}

Not applicable.

\section{CONFLICT OF INTEREST}

The authors declare no conflict of interest, financial or otherwise.

\section{ACKNOWLEDGEMENTS}

Declared none.

\section{REFERENCES}

[1] Deming W. The New Economics for Industry, Government, and Education. Cambridge, AM: MIT Press 2000.

[2] Edwards PJ, Huang DT, Metcalfe LN, Sainfort F. Maximizing your investment in EHR. Utilizing EHRs to inform continuous quality improvement. J Healthc Inf Manag 2008; 22(1): 32-7. [PMID: 19267005]

[3] Health Resources and Services Administration Quality Improvement April 2011. Available at: https:/www.hrsa.gov/quality/toolbox/508pdfs/qualityimprovement.pdf 
[4] Jung C, Ed. Psychological Types, Collected Works of CG Jung. Princeton, NJ: Princeton University Press 1971.

[5] Clack GB, Allen J, Cooper D, Head JO. Personality differences between doctors and their patients: Implications for the teaching of communication skills. Med Educ 2004; 38(2): 177-86. [http://dx.doi.org/10.1111/j.1365-2923.2004.01752.x] [PMID: 14871388]

[6] Association of American Medical Colleges Core Entrustable Professional Activities for Entering Residency: Faculty and Learners' Guide 2014. Available at: https://members.aamc.org/eweb/upload/Core\%20EPA\%20Faculty\%20and\%20Learner\%20Guide.pdf

[7] Myers IB, McCaulley MH, Quenk NL, Hammer AL, Eds. MBTI manual: A guide to the development and use of the Myers-Briggs Type Indicator. 3rd ed. Palo Alto, CA: Consulting Psychologists Press 1998.

[8] Allen J, Brock SA, Eds. Health care communication using personality type: patients are different!. London: Routledge 2000.

[9] Spencer LM, Spencer SM. Competence at work: Models for superior performance. New York: John Wiley \& Sons 1993.

[10] Heng HH. The conflict between complex systems and reductionism. JAMA 2008; 300(13): 1580-1. [http://dx.doi.org/10.1001/jama.300.13.1580] [PMID: 18827215]

[11] Senge P. The fifth discipline: The art and practice of the learning organization. New York: Doubleday/Currency 1990.

[12] Tuckman BW. Developmental Sequence in Small Groups. Psychol Bull 1965; 63: 384-99. [http://dx.doi.org/10.1037/h0022100] [PMID: 14314073]

[13] Lawrence G. People Types and Tiger Stripes. $3^{\text {rd }}$ ed. Gainesville, FL: Center for Applications of Psychological Type 1993.

[14] Ericsson KA. Acquisition and maintenance of medical expertise: A perspective from the expert-performance approach with deliberate practice. Acad Med 2015; 90(11): 1471-86. [http://dx.doi.org/10.1097/ACM.0000000000000939]

[15] Ricard M, Lutz A, Davidson RJ. Mind of the meditator. Sci Am 2014; 311(5): 38-45. [http://dx.doi.org/10.1038/scientificamerican1114-38] [PMID: 25464661]

[16] , Eds. Levy DM, Wobbrock JO, Kaszniak AW, Ostergren M, Eds. The effects of mindfulness meditation training on multitasking in a highstress information environment. GI 12 Proceedings of Graphic Interface; 2012 May 28-30, Toranto, Ontario, Canada.

[17] Gorman TE, Green CS. Short-term mindfulness intervention reduces the negative attentional effects associated with heavy media multitasking. Sci Rep 2016; 6: 24542. [http://dx.doi.org/10.1038/srep24542] [PMID: 27086504]

[18] Benson H. The Relaxation Response. New York: William Morrow 1975.

[19] Keng SL, Smoski MJ, Robins CJ. Effects of mindfulness on psychological health: a review of empirical studies. Clin Psychol Rev 2011; 31(6): 1041-56

[http://dx.doi.org/10.1016/j.cpr.2011.04.006] [PMID: 21802619]

[20] Leigh J, Bowen S, Marlatt GA. Spirituality, mindfulness and substance abuse. Addict Behav 2005; $30(7): 1335-41$. [http://dx.doi.org/10.1016/j.addbeh.2005.01.010] [PMID: 16022930]

[21] Zeidan F, Grant JA, Brown CA, McHaffie JG, Coghill RC. Mindfulness meditation-related pain relief: Evidence for unique brain mechanisms in the regulation of pain. Neurosci Lett 2012; 520(2): 165-73. [http://dx.doi.org/10.1016/j.neulet.2012.03.082] [PMID: 22487846]

C 2017 Peck and Pelley.

This is an open access article distributed under the terms of the Creative Commons Attribution 4.0 International Public License (CC-BY 4.0), a copy of which is available at: https://creativecommons.org/licenses/by/4.0/legalcode. This license permits unrestricted use, distribution, and reproduction in any medium, provided the original author and source are credited. 